\title{
Las protestas de los ciudadanos. Características y peculiaridades de la movilización territorial en el Véneto
}

\author{
Laura Fregolent \\ Università IUAV di Venezia. Dipartimento di Progettazione \\ e Pianificazione in Ambienti Complessi \\ laura.fregolent@iuav.it
}

Recepción: julio de 2012

Aceptación: noviembre de 2014

\section{Resumen}

Este trabajo presenta los resultados de una investigación en la que se destaca el gran número y variedad de conflictos relacionados con las transformaciones territoriales que hoy existen en el Véneto. El elevado número de conflictos y comités activos indica un interés constante por las cuestiones medioambientales y además pone de relieve el importante rol que han jugado las dinámicas de desarrollo y uso del territorio — surgidas en esta región en las últimas décadas - en el fenómeno que ahora se va manifestando.

Esta conflictividad se debe interpretar no sólo como la demanda de los ciudadanos para participar y estar más implicados en los procesos de transformación territorial —que repercuten directamente en ellos y en la calidad de vida-, sino también como su declarada falta de confianza en una clase política a menudo incapaz de manejar los conflictos y por tanto «débil» para planificar y hacerse cargo de situaciones conflictivas.

Palabras clave: conflictos territoriales; participación ciudadana; planificación.

Resum. Les protestes dels ciutadans. Caracteristiques i peculiaritats de la mobilització territorial a la regió del Véneto

Aquest treball presenta els resultats d'una investigació en què s'ha destacat el gran nombre i varietat de conflictes relacionats amb les transformacions territorials que existeixen avui dia a la regió del Véneto. La quantitat de conflictes i d'associacions actives demostra l'interès constant per les qüestions mediambientals i també posa en relleu el paper central que han jugat les dinàmiques de desenvolupament i ús del territori — presents a la regió en les últimes dècades - en el fenomen que ara s'està manifestant.

Els conflictes s'han d'interpretar no tan sols com a resultat de la demanda dels ciutadans per participar i influir més en els processos de transformació territorial — que hi repercuteixen directament i en la qualitat de vida—, sinó també com a resultat de la seva declarada falta de confiança en una classe política sovint incapaç de gestionar els conflictes i per tant «feble» per planificar i fer-se càrrec de situacions conflictives.

Paraules clau: conflictes territorials; participació ciutadana; planificació. 
Résumé. Les protestations des citoyens. Caractéristiques et particularités de la mobilisation territoriale dans la Vénétie

L'article évalue les résultats de la recherche à travers laquelle ont été mis en évidence le nombre et les formes de conflits territoriaux actuels dans la région de la Vénétie. Le nombre de conflits et d'associations actives montre un intérêt constant concernant les questions environnementales, et met en évidence la manière dont la dynamique de l'aménagement du territoire et de l'utilisation des terres - produites dans la région de la Vénétie lors des dernières décennies - ont joué un rôle important dans le phénomène qui se manifeste aujourd'hui.

Le conflit doit être lu comme une demande de participation, d'implication dans les processus territoriaux qui ont un impact direct sur les citoyens et la qualité de vie. Il est également une déclaration de manque de confiance envers une classe politique qui a perdu progressivement sa capacité à gérer les conflits.

Mots-clé: conflits territoriaux; participation des citoyens; planification.

Abstract. Public protests. Characteristics and peculiarities of territorial conflicts in the Veneto region

This paper shows the results of a research project on territorial conflicts in the Veneto region. The research describes the various types of conflict connected with territorial transformations. The number of conflicts and active committees suggests a lifelong interest in environmental issues as well as highlighting the significant role that land development and land use dynamics - which have occurred in the Veneto region in recent decades - are playing in this new phenomenon.

Conflicts should be interpreted not only as a public demand for more participation and involvement in territorial processes that have a direct impact on them and their standard of living, but also as a self-professed lack of confidence in a political class that has gradually reduced the capacity for political representation.

Keywords: territorial conflicts; public participation; urban planning.

\section{Sumario}

Objetivo, motivaciones y metodología Antecedentes y posibles claves para su lectura

El caso de estudio Conflictos y comités
El origen del conflicto: la falta de planificación

Conclusiones

Referencias bibliográficas 


\section{Objetivo, motivaciones y metodología}

Este trabajo presenta los resultados de una investigación llevada a cabo por el Istituto Universitario di Architettura de Venecia, en colaboración con Legambiente Veneto, que tenía por objetivo elaborar un Atlas del malestar territorial ${ }^{1}$ a partir de una base de datos donde obras y proyectos contestados socialmente se encuentran catalogados a través de fichas y mapas de localización ${ }^{2}$. De él se desprende el gran número y variedad de conflictos relacionados, sobre todo, con decisiones tomadas en la planificación territorial y, por consiguiente, con las transformaciones territoriales que existen en la región del Véneto. Situada en el noreste de Italia, se trata de una región que ha experimentado, a lo largo de los últimos decenios, un extraordinario desarrollo económico y una transformación territorial, alguna de cuyas consecuencias son los conflictos de los que se va a tratar en este artículo.

Los datos se han recogido gracias también a la ayuda de comités de ciudadanos, asociaciones ambientalistas y de otro cariz social, que en algún momento se han movilizado contra las intervenciones de transformación territorial. La participación de estos comités y asociaciones ha hecho posible recopilar información sobre el origen, su evolución del conflicto y los distintos actores implicados, y ha permitido explicar las motivaciones y las expectativas de estos actores.

Las razones que han llevado a la realización de esta base de datos son diferentes. A lo largo de los últimos años - y no sólo en Italia — se ha observado un creciente fenómeno de microconflictividad, como si se tratara de una nueva manera de asistir a los debates, de participar en la toma de aquellas decisiones que implican transformaciones en el territorio. Ha resultado muy interesante sistematizar este fenómeno y, además, colaborar, por medio de instrumentos de recopilación y catalogación, en la monitorización de una casuística que va en aumento. Finalmente, se ha intentado comprobar si existe un hilo conductor entre las distintas manifestaciones analizadas, entre los proyectos y las opciones urbanísticas que han provocado las movilizaciones de protesta, a partir del análisis de las obras realizadas y de su impacto en las comunidades locales.

El mapa de los conflictos (Figura 1) es amplio y complejo, debido a que son muchas las razones de protesta y muchos los actores implicados. Los conflictos se pueden producir entre los ciudadanos y la administración pública (ayuntamiento, provincia, región, estado, etc.); por proyectos que tienen un sensible impacto o provocan problemas y malestar entre la población (construcción de nuevas infraestructuras, instalaciones de tratamiento y/o eliminación de residuos, etc.); entre grupos de ciudadanos, asociaciones y privados, debido a la realización de obras que ponen en peligro el paisaje y el medio ambiente; entre distintas administraciones públicas por decisiones que se han tomado en

1. Para ello, se ha tomado como referencia el Anuari territorial de Catalunya.

2. A este respecto véase: $<$ http://mapserver.iuav.it/website/AtlanteMalessereTerritoriale/AtlanteMalessereTerritoriale.html.>. 


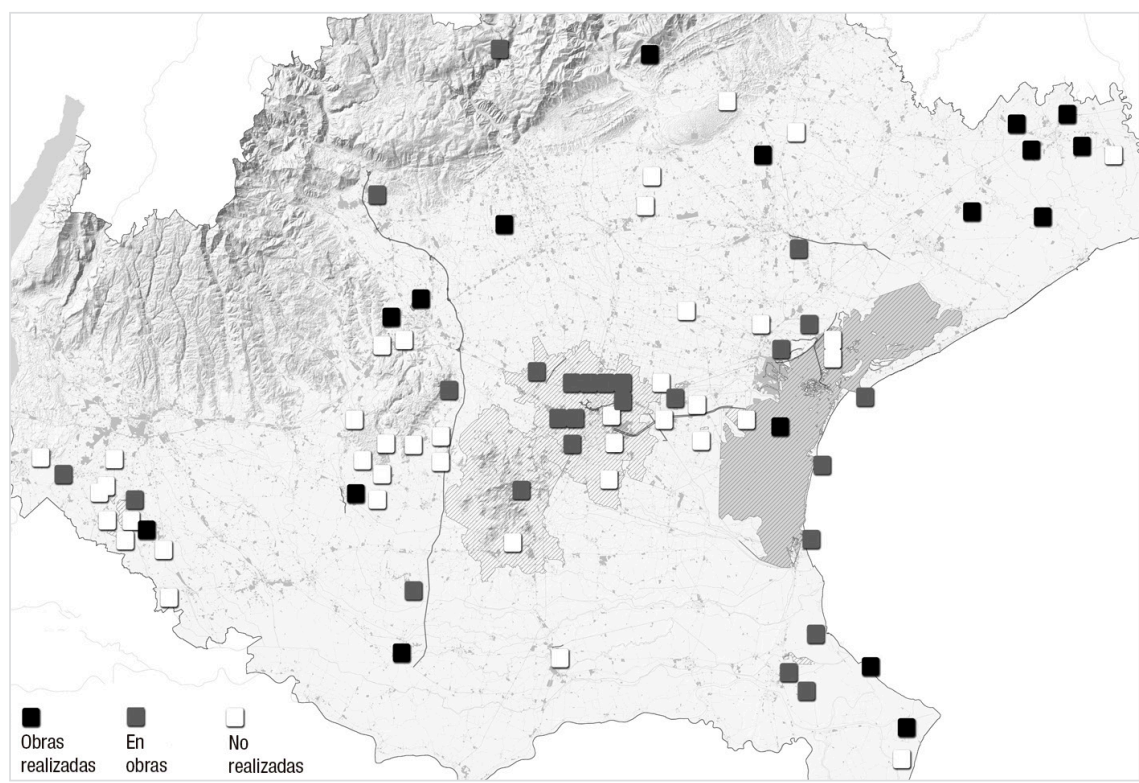

Figura 1. Mapa de los conflictos provocados por: obras realizadas (15); en curso (25); no realizadas (31).

Fuente: <http://mapserver.iuav.it/website/AtlanteMalessereTerritoriale/AtlanteMalessereTerritoriale.html>. Elaborado por Giuliana Fornaciari.

un determinado nivel institucional pero que tienen repercusiones «negativas» en otro, etc.

Las movilizaciones analizadas nos ayudan a trazar un interesante cuadro general, no sólo en relación al gran número de obras que originan protestas, sino también en cuanto a la falta de cualquier tipo de control por lo que se refiere a las transformaciones territoriales que han caracterizado los últimos cuarenta años en la región. Estas movilizaciones tienen un marcado carácter contestatario y reivindicativo (Vitale, 2007: 12) contra algunas opciones concretas que han llevado a realizar en el territorio obras de alto impacto ambiental y cuyas consecuencias negativas afectan a la calidad de vida de las poblaciones allí asentadas.

Uno de los rasgos de estas movilizaciones es, por lo tanto, la conflictividad. Como es sabido, todo conflicto refleja la variedad social y los distintos intereses y posturas en juego. En este sentido, se convierte en un ejercicio de democracia y, a veces, en la evolución de un proceso que define una posible modalidad de relación entre las partes. Sin embargo, también puede suponer malestar e inquietud, e indicar que se ha roto el diálogo entre los que deciden y mandan a nivel político y los que preconizan la necesidad de un cambio. En este caso, se puede hablar de un proceso democrático que se ha interrumpido evidenciando la urgencia de reconsiderar las soluciones adoptadas para buscar una alternativa viable. 


\section{Antecedentes y posibles claves para su lectura}

El número creciente de conflictos territoriales (SCOT, 2007; Bobbio, 2011; Cruz y Martí, 2010; San Martín, 2012) impone algunas reflexiones sobre el porqué las comunidades locales participan cada vez más en la defensa de "su» territorio (Bobbio y Zeppetella, 1999; Nel-lo, 3003; Pellizzoni, 2011), es decir, cuáles son las causas de este fenómeno que se muestra —en sus distintas facetas y manifestaciones locales - cada vez más extendido.

En efecto, se puede examinar el fenómeno desde una perspectiva global y no solamente desde la conflictividad ambiental o territorial (Della Porta y Tarrow, 2005; Castells, 2012). De hecho, nos enfrentamos no sólo a un aumento progresivo de las temáticas de las protestas — que ya han dejado de ser sólo ambientales-, sino también a reivindicaciones que intentan involucrar a las cuestiones relacionadas con la defensa del territorio, del paisaje (Nogué y Wilbrand, 2010), del patrimonio natural y artístico, siempre desde el marco de la defensa de un bien común y colectivo.

Bobbio (2011) identifica seis posibles «respuestas» al porqué del número creciente de conflictos y expone también cuáles son las acciones que se pueden acometer, partiendo del principio que no existe, justamente, una respuesta única ni respecto a las motivaciones ni a las causas que ocasionan conflictos, ni tampoco respecto a las posibles acciones que se pueden emprender.

La primera subraya la inclinación local de los movimientos y los adscribe al fenómeno NIMBY; la segunda incide en la instrumentalización de la protesta con vistas a otras finalidades; la tercera evalúa el conflicto como una consecuencia del desequilibrio entre costes y beneficios procedentes del asentamiento propuesto, ya que los costes gravan sobre la comunidad a la que le corresponde afrontar los gastos, en cambio los beneficios, a veces, van a parar a quien no vive necesariamente en ese entorno (Bobbio, 1994); la cuarta se centra en el riesgo relacionado con las consecuencias del asentamiento propuesto; la quinta, en cambio, hace referencia a un potencial riesgo para las comunidades asentadas, partiendo de la relación entre "lugares» $\mathrm{y}$ «flujos» evidenciada por Castells (1983) y de la oposición generada por los sentimientos de identidad local. Por último, la sexta, identifica en las más recientes contestaciones una común y explícita reacción ante el modelo dominante de desarrollo (Rootes, 2005; Della Porta y Piazza, 2008; Fedi y Mennarini, 2008; Caruso, 2010), reacción que se aleja de la actitud NIMBY y que vuelve a considerar las motivaciones de los movimientos desde una perspectiva más amplia.

Los grupos afectados, en efecto, critican duramente a las autoridades y las decisiones tomadas por éstas respecto a su territorio: acusan a los funcionarios, partidos políticos y políticos sin más de ser responsables del deterioro del medio ambiente, por lo que la protesta de los comités adquiere el carácter de la "participación contra» (Rosanvallon, 2006) y se convierte en un instrumento que permite situarse en el centro del debate y de los procesos decisorios.

Sin duda, las seis hipótesis formuladas facilitan también una lectura del caso aquí propuesto y de la interpretación de las dinámicas y de las motivaciones 
que desencadenan las protestas, pero uno de los objetivos es el de averiguar cómo y de qué manera la proliferación de los conflictos se debe a una «débil» o ausente planificación «de les polítiques territorials — d'infraestructures, energia, equipaments, urbanisme - aplicades des de l'Administració; polítiques que sovint estan mal dissenyades i gairebé sempre mal explicades» (Nel.lo, 2003: 12); es decir, una planificación que no fomenta la participación activa del ciudadano en la elección de los planes (Cruz y Gallach, 2009) desencadena la formación de comités de protesta.

Sería necesario, en cambio, construir una nueva relación entre planificación y conflicto, en la que el conflicto, además de asumir su papel en la formación de las «identidades colectivas» (Fainstein, 2005; Della Porta, 2008), se convierta también en un recurso, ya que la innovación no se produce en presencia de un reparto homogéneo de objetivos y valores, sino en una área intermedia y de oposición, una «zona de intercambio» limitada; precisamente una "trading zone», que permita «innovaciones espaciales que se pueden adscribir a estrategias — en conflicto incluso entre ellas - y que, a diferencia «de los planteamientos participativos, no determina el prevenirlos o gestionarlos, sino afrontarlos en profundidad para comprender qué es lo que, en todo caso, se puede hacer aunque sigamos siendo enemigos» (Balducci, 2013: 125-126).

Todo esto afecta a las políticas urbanas e, igualmente, a la planificación en su totalidad, planificación que podría adquirir un papel fundamental en la prevención/solución de los conflictos relacionados con obras indeseadas. Sin embargo, para poder hacer esto, haría falta un planning research «to return to the concrete, to the empirical and to case research, not as a mindless return to empiricism, but as a way of gaining a better understanding of the nature of difference, and generating ideas and propositions which can more adequately inform practice» (Watson, 2003: 396).

\section{El caso de estudio}

La recopilación y elaboración de los datos ha conseguido trazar un cuadro general bastante amplio de la situación regional. Se han registrado en total 72 casos de conflictos concentrados principalmente en el Véneto central (Figuras 1 y 2).

La primera constatación a destacar se refiere, por lo tanto, al elevado número de conflictos presentes en el territorio a pesar de que la recopilación aún no se ha concluido, ya que quedan todavía por registrar algunas movilizaciones que en los últimos tiempos han ido adquiriendo más relevancia.

La primera elaboración de los datos se refiere a los conflictos detectados y los divide en grupos según la actuación específica que ha llevado a la movilización colectiva. De los 72 registrados, 58 siguen abiertos y 14 de ellos se han dado por finalizados. Se trata de conflictos de diferentes dimensiones e intensidades que se pueden clasificar como sigue (Tabla 1).

Se trata de obras de alto impacto ambiental (sobre todo en el caso de las carreteras u obras infraestructurales) que van a fragmentar el tejido urbano, que en toda el área del Véneto central se caracteriza por asentamientos dispersos en 


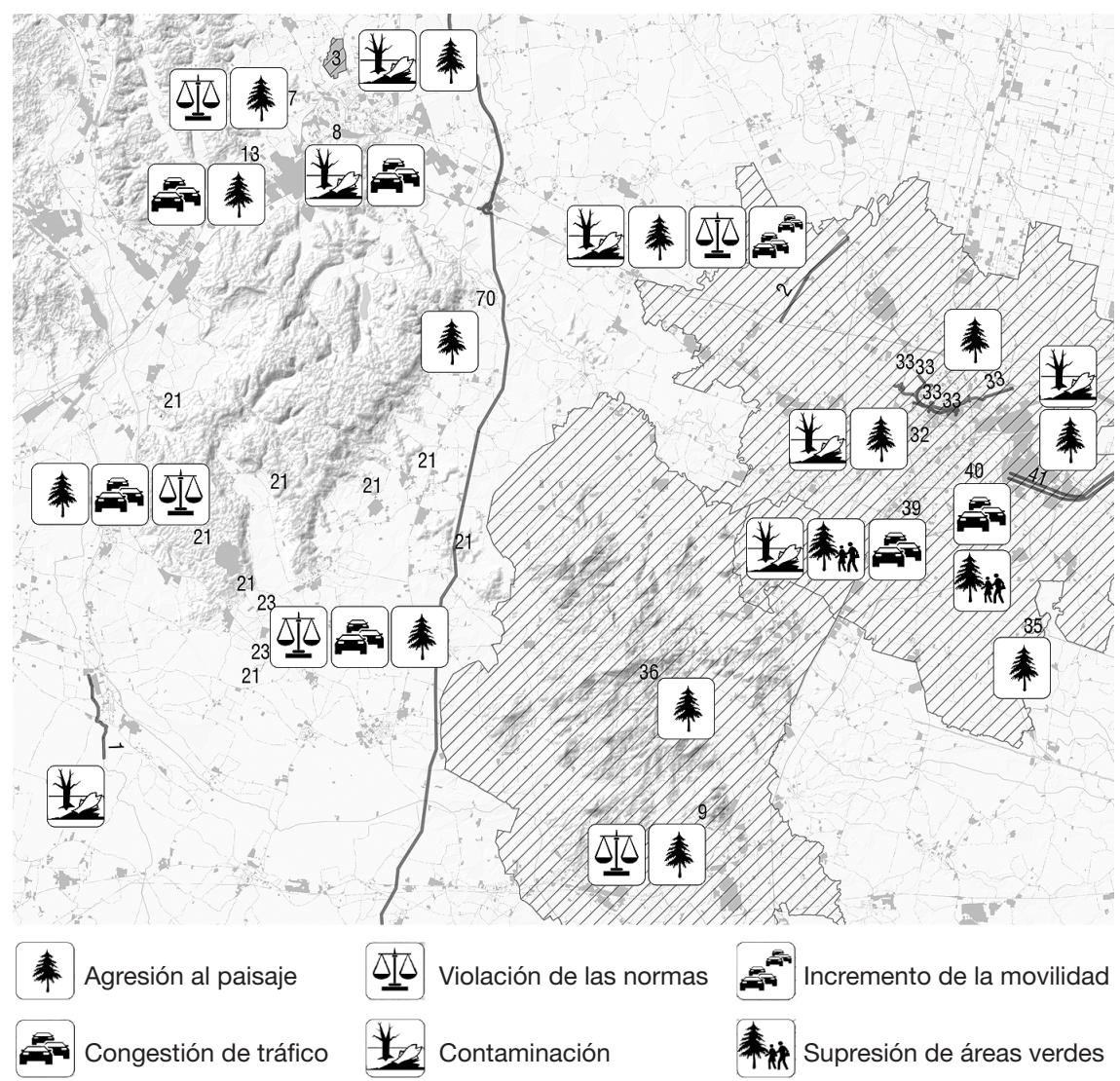

Figura 2. Causas «subyacentes».

Fuente: <http://mapserver.iuav.it/website/AtlanteMalessereTerritoriale/AtlanteMalessereTerritoriale.html>. Elaborado por Giuliana Fornaciari.

Tabla 1. Causas de los conflictos

Infraestructuras e instalaciones relacionadas

Instalaciones de eliminación y tratamiento de residuos

Fomento de las actividades productivas

Viviendas, establecimientos comerciales y turísticos

Producción de energía

Vertidos de sustancias contaminantes

Fuente: <http://mapserver.iuav.it/website/AtlanteMalessereTerritoriale/AtlanteMalessereTerritoriale.html>. 


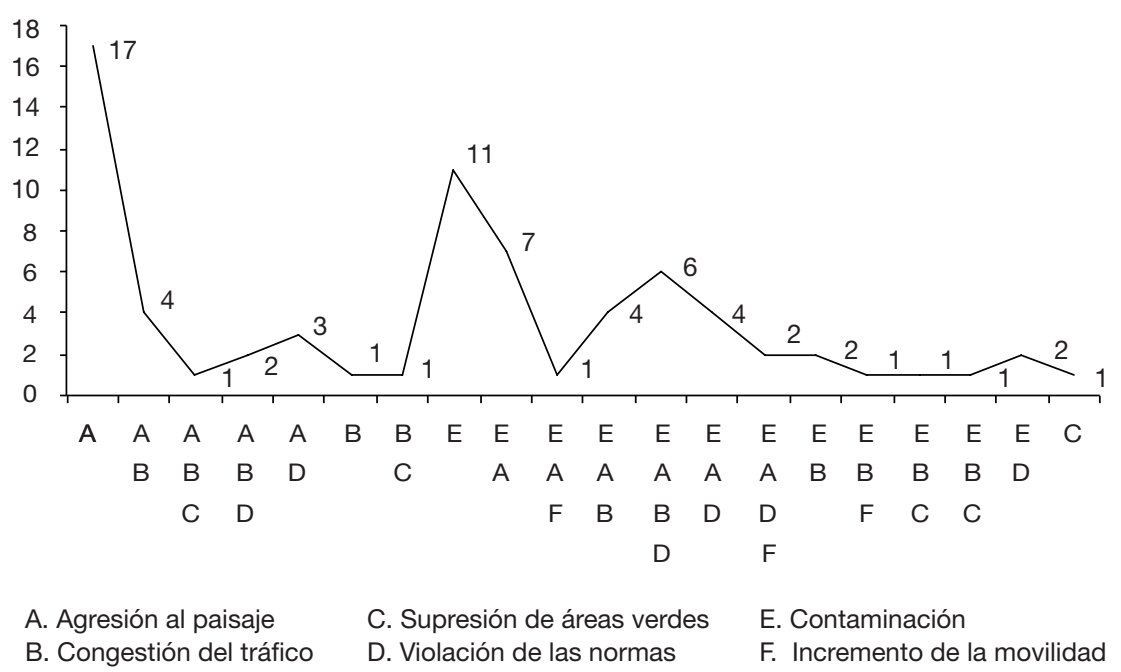

Figura 3. Causas «subyacentes» relacionadas con distintos conflictos, según el número de conflictos.

Fuente: <http://mapserver.iuav.it/website/AtlanteMalessereTerritoriale/AtlanteMalessereTerritoriale.html>.

su forma y estructura pero conectados desde el punto de vista de las relaciones sociales; o bien de proyectos, a veces de grandes dimensiones (algunos de ellos en fase de realización). Estos últimos amenazan el paisaje e incrementan el consumo de suelo libre, lo que provoca un alto impacto tanto ambiental como social debido a que abarcan áreas bastante amplias, acarrean consecuencias a nivel de movilidad y representan un riesgo para algunas economías locales, como en el caso de intervenciones de tipo comercial. Las mismas consideraciones se pueden hacer por lo que se refiere a los conflictos relacionados con excavaciones o proyectos de fuerte impacto en áreas verdes que los planes urbanísticos ya habían destinado a parque urbano y que, sin embargo, con el paso del tiempo se han vuelto más apetecibles para los inversores debido a su localización y potencial de transformación. Lo que se pretendería sería convertirlas en áreas urbanizables, prescindiendo por completo tanto de la importancia de los espacios verdes en los barrios residenciales y de su función de pulmón verde, como del propio derecho de los ciudadanos a tener una calidad de vida que mucho tiene que ver, entre otras cosas, con la calidad de los espacios verdes públicos. Por consiguiente, las protestas se dirigen contra un tipo de actividad que supone una agresión irreparable a los valores paisajísticos y ambientales.

Menos numerosas resultan ser las protestas relacionadas con la cuestión de los residuos, desde su tratamiento a la limpieza de suelos contaminados, pasando por la ampliación de un vertedero de residuos no peligrosos. Cabe subrayar que la cuestión del tratamiento de los residuos sólidos urbanos se ha ido modi- 
ficando a lo largo de las últimas dos décadas. Efectivamente, la introducción de medidas de seguridad en los vertederos, la aplicación gradual de normas sobre el reciclaje y la reutilización de los materiales de desecho han proporcionado una solución casi definitiva a este problema. Por lo tanto, las antiguas protestas relacionadas con la instalación de nuevos vertederos o ampliar los ya existentes, hoy en día se centran más bien en la gestión de residuos peligrosos y en la creación de instalaciones para el tratamiento de los residuos orgánicos y la producción de biogás.

La clasificación de los conflictos a partir del tipo de proyectos con los que están relacionados evidencia su causa principal, desencadenada por la realización de una obra o una transformación territorial: carreteras, infraestructuras, contaminación, expansiones residenciales o de otro tipo, residuos, excavaciones, áreas verdes, planes de área. A éstas hay que añadir otras causas que podríamos llamar «subyacentes» (contaminación; agresión al paisaje; incumplimiento de normas; congestión del tráfico; eliminación de áreas verdes; etc.) y que agudizan aún más los conflictos existentes (Figuras 2 y 3 ). Quienes han elaborado las fichas han interpretado estas causas no sólo como razones del conflicto, sino también como efectos del proyecto o de la obra ya realizada o por realizar. Resumiendo: las protestas se caracterizan por una causa principal que se identifica con el propio conflicto y por muchas otras con las que mantiene una estrecha relación.

Así pues, un conflicto nunca depende de una causa aislada, sino de varios factores estrechamente relacionados con el contexto en que se inscribe la obra a realizar (fábricas asentadas en áreas con restricciones ambientales, por ejemplo) y a veces con cuestiones específicas: la propia realización de la obra, las modalidades de la toma de decisiones, los motivos del enfrentamiento entre los actores implicados (Bobbio, 2011). Además, a la causa desencadenante, que es la razón principal del conflicto (la realización de la obra, la alteración sustancial del proyecto inicial, la contaminación y los riesgos para la salud) se suman otros factores, cuya identificación permite analizar el fenómeno más detalladamente y aportar eventuales correcciones al proyecto para limitar su impacto.

Entre los factores que pueden estar relacionados con la causa del conflicto destacan los siguientes:

- el grado de irreversibilidad alcanzado por todas las obras infraestructurales (y, más en general, por cualquier obra) que estén destinadas a tener un impacto espacial que se pueda revelar como una «agresión al paisaje»;

- la crisis de las formas de representación;

- la escasa predisposición a colaborar que suele haber entre los distintos niveles de gobierno;

- la incapacidad de un administrador local para gestionar las transformaciones territoriales de alto impacto cuya realización se haya decidido a un nivel de gobierno más alto;

- la respuesta del poder supralocal que a menudo no tiene suficientemente en cuenta la dimensión local; 
- la densidad de los asentamientos y la nueva morfología del territorio que, sobre todo en algunas áreas de la región, dificulta la localización de edificios, nuevas infraestructuras, etc.;

— una información pública tardía que comunica al ciudadano la realización de la obra sólo cuando ésta ya se inicia;

- la Administración Pública que, desempeñando varios papeles, corre el riesgo de incurrir en un conflicto de intereses;

- la escasa predisposición a la transparencia, tanto en los procesos y modalidades de organización y autorización de la obra como en la toma de decisiones;

- la tendencia a implicar a los diversos actores en los procesos de transformación territorial, sea que tengan intereses económicos, sea que se trate de asociaciones o de simples ciudadanos (cfr. Bartolomeo, 2009); y, finalmente,

— la falta de políticas territoriales (Nel.lo, 2003).

\section{Conflictos y comités}

En el Véneto, el número de comités ha ido creciendo a lo largo del tiempo. De un estudio realizado por el Observatorio sobre las Transformaciones en el Véneto a finales de los noventa (Zamparutti, 2000), los comités resultaron ser más de cien. En 2007, el Departamento de Geografía de la Universidad de Padua se encargó de poner al día los datos de las provincias de Treviso, Padua y Vicenza, e indicó que los 72 comités de 1998 habían pasado a ser 140, es decir, el doble de los registrados la primera vez (Varotto y Visentin, 2008).

Los comités y delegaciones de asociaciones ecologistas que han participado o han estado implicados en las protestas son unos 90 . No se incluyen los partidos políticos cuya implicación directa es muy limitada, si se exceptúa la participación esporádica de Lega, Verdi, Sinistra Ecologia e Libertà y Federazione della sinistra, es decir, por un lado, las fuerzas que están más a la izquierda del arco constitucional —y que históricamente han estado más implicadas en las luchas ecologistas - y, por otro, la Lega, un partido con un fuerte arraigo territorial y un fuerte sentido identitario.

No siendo el objetivo principal del estudio el de averiguar el número de comités existentes, el resultado posiblemente sea parcial, pero, a pesar de ello, nos proporciona algunas indicaciones: en las protestas, junto con comités de inspiración diversa, comités ligados al ecologismo, grupos de ciudadanos residentes en los pueblos afectados, asociaciones de padres, etc., todos ellos, caracterizados por un fuerte espontaneísmo, participan en asociaciones ecologistas históricas y más organizadas tales como Legambiente, Italia Nostra, WWF y Lega Italiana Protezione Uccelli (LIPU: liga italiana para la protección de las aves).

El hecho de que estas asociaciones históricas, reconocidas por el Ministerio de Medio Ambiente, participen en la protesta, tanto si están directamente implicadas como si apoyan una acción promovida por otros comités, nos lleva a reflexionar sobre la esencia del conflicto y sus modalidades: muchas veces 
las protestas se van apagando poco a poco porque falta una estructura capaz de organizarlas, coordinarlas y sobre todo mantenerlas vivas; en cambio, las asociaciones ecologistas - $\mathrm{y}$ en general todas las organizaciones estructuradasaseguran continuidad a la acción colectiva (Della Porta y Diani, 1997). Esto no necesariamente vale para los casos analizados: no podemos averiguar si las protestas en que han participado las asociaciones históricas han sido más incisivas y organizadas que las otras, pero sabemos, según indican algunas fichas, que para los pequeños comités es más duro y difícil mantener vivas las protestas.

Por ejemplo, según la ficha de la protesta contra la ampliación de la base de la OTAN en Vicenza, la puesta en marcha de las obras a mediados de 2009, la sentencia dictada por el Juzgado Administrativo Regional (TAR) que, desde el punto de vista jurídico, cierra definitivamente la cuestión y el cambio de postura de la administración municipal, han acabado por poner al movimiento en una condición de debilidad en la que resulta más difícil o imposible emprender nuevas iniciativas o traducir la protesta en una acción política concreta. Lo único bueno de la nueva situación es que en el mes de junio de 2010 se decidió desmilitarizar y sustraer del dominio público estatal una amplia área junto al antiguo aeropuerto para convertirla en un gran parque urbano, aunque todavía no están claros los aspectos burocráticos y financieros de la cuestión. Esta representa sin duda una victoria que el Movimiento logró gracias a las buenas relaciones establecidas entre ciudadanos y administración local y al apoyo de las fuerzas que constituyen la mayoría política en el seno de la corporación municipal. La idea del parque basada en una propuesta de resolución presentada por iniciativa popular es, de momento, el único ejemplo de convergencia entre los diversos sectores del Movimiento, que ahora tendrían que demostrar su capacidad para buscar estrategias comunes y para realizar acciones de participación conjunta y de control constante. Los movimientos tienen, por lo tanto, dificultades objetivas que los llevan a desarticularse o por lo menos a incidir solo hasta cierto punto; o incluso pueden llegar a desaparecer del todo, como en el caso de la terminal de gas de Porto Viro en el delta del Po (cfr. Amistani, 2011: 149).

El aumento de comités y protestas ha sufrido, a lo largo del tiempo, transformaciones ligadas a los fenómenos territoriales que se estaban desarrollando. Mientras que en los años noventa las causas de las protestas estaban relacionadas con el tratamiento de residuos (nuevos vertederos o problemas de gestión de antiguos vertederos, termovalorizadores y contaminación provocada por la eliminación de residuos) y la realización de nuevas infraestructuras, ahora atañen, por lo general, a la preservación de la naturaleza y del paisaje, oponiéndose a todo tipo de opción que implique una transformación del uso y consumo del suelo (Della Porta y Diani, 2004).

Los casos analizados constatan que las movilizaciones actuales están causadas, en su mayoría, por cuestiones ligadas a la realización de infraestructuras y a la agresión al territorio y paisaje, aunque quedan ejemplos de protesta más «tradicional» no sólo contra las descargas industriales cuyos vertidos acaban en los cauces de los ríos y contra el tratamiento de residuos líquidos especiales 
en barrios densamente poblados, sino también contra los proyectos para la adecuación tecnológica y las mejoras técnicas en las instalaciones industriales. Estas últimas siempre han constituido un tema muy controvertido y relevante: si para las asociaciones ambientalistas representan un riesgo de impacto ambiental, para los sindicatos representan puestos de trabajo, lo que ha llevado a la fuerte yuxtaposición entre las partes y, por consiguiente, a que sea más difícil encontrar soluciones rápidas a los problemas.

La relación entre ocupación laboral y protección del territorio nos lleva también a reflexionar sobre las oportunidades que algunas obras pueden representar desde el punto de vista económico y laboral. Esto se refiere no sólo a las infraestructuras, que desde siempre se consideran como un trampolín para los procesos de transformación tanto del territorio como del sector económico, sino también a las expansiones productivas, a los centros comerciales y a los proyectos de gran transformación territorial, aunque no siempre es fácil medir el retorno de las inversiones y las ventajas que representan para la colectividad.

La adhesión a movimientos y comités de protesta se debe interpretar como una forma de participación activa, de implicación en los procesos de transformación territorial, de voluntad de influir en las decisiones que repercuten directamente en los ciudadanos y su calidad de vida. Estos movimientos pueden implicar a un número variable de personas que tienen una razón común para protestar. Las cuestiones tratadas no son estrictamente locales y particulares, es decir, reconducibles del todo a los fenómenos NIMBY (Bobbio, 2011), aunque las protestas a escala nacional de los noventa no estaban exentas de ellos (Della Porta y Diani, 2004); parece ser que los comités representan la voluntad de participar en el gobierno del territorio, si bien no están lo suficientemente politicizados como para salir del ámbito local y llegar a tener mayor alcance.

Además, las acciones colectivas orientadas a la defensa de derechos o a la producción de bienes públicos constituyen un «factor de consolidación de sentimientos más generales de adhesión a una sociedad civil ya que puede transformar la desconfianza y la rabia en propuestas y acciones dirigidas a la esfera pública. De esta manera las asociaciones de voluntarios pueden contribuir a reproducir, a partir de nuevas bases, una relación de lealtad e identificación con las instituciones y la comunidad política, en un momento en que a los partidos políticos se los consideraba en larga medida deslegitimados» (Biorcio, 2008).

Todo ello significa que las formas de la participación democrática evolucionan: frente a la pérdida de importancia y función de los partidos, a las que corresponde un fuerte abstencionismo electoral, va tomando pie el asociacionismo de los ciudadanos que, además del voto, tienen otro instrumento útil para manifestar su disconformidad con quien gobierna (Rosanvallon, 2006).

Los comités suelen estar muy bien informados y bastante organizados, conocen muy bien el contexto territorial en que se mueven y los problemas o perjuicios que una determinada obra puede acarrear, entienden de política y por lo general identifican posibles soluciones alternativas, aunque en realidad las protestas de carácter político que inciden en la totalidad del territorio y que tienen perspectivas de «sostenibilidad» son más limitadas. Esta es, en cambio, 
una prerrogativa de las asociaciones ecologistas con una fuerte organización o de algunos grupos de coordinación que han emprendido acciones colectivas, tal como la protesta contra el Plan Territorial Regional de Coordinación de Véneto. En aquella ocasión varios comités reunidos en un grupo de coordinación regional se movilizaron y elaboraron un documento crítico sobre el Plan que respondía a la fuerte voluntad política de participar e influir en la gestión de los bienes públicos en nombre del interés general, ese mismo interés que, a veces, los que deberían administrar los asuntos públicos pierden de vista a la hora de identificar los objetivos y poner en práctica las decisiones tomadas previamente.

\section{El origen del conflicto: la falta de planificación}

Las cuestiones relacionadas con la destrucción del paisaje y la mala gestión del territorio tienen mucho que ver con las opciones de planificación de los diversos niveles de gobierno y vuelve a poner en discusión las políticas urbanísticas de región, provincias y ayuntamientos por los instrumentos de planeamiento que adoptan y las decisiones que toman, cada uno en su ámbito de responsabilidad.

A menudo, los proyectos y obras que originan protestas no respetan los instrumentos de la planificación vigentes, y se desvirtúan con las modificaciones ad hoc, necesarias para que las obras se puedan realizar. El caso del autódromo del Véneto ${ }^{3}$, en la provincia de Verona, es un ejemplo típico de esta conducta -El Plan de Área Quadrante Europa (10/1999) indicaba una zona de 100 hectáreas donde realizar el circuito del autódromo del Véneto y sus infraestructuras, aunque protegía la mayor parte de este territorio por su interés paisajístico y ambiental y por estar ya destinado a formar parte del parque fluvial de la llanura de Verona.

En 2003, los ayuntamientos implicados se apresuraron a introducir una modificación en el Plan Director Municipal (PRG) y la Junta Regional adoptó una serie de medidas y variantes para añadir al proyecto nuevas funciones comerciales sin poner límites a las dimensiones de las obras y reduciendo el porcentaje de áreas permeables. En definitiva, el proyecto del autódromo que inicialmente se inscribía en un contexto protegido y a preservar en el futuro, se convirtió en una ocasión de desarrollo que aprovechar sin mayores impedimentos.

Ejemplos de este tipo son muy frecuentes tanto a escala territorial, gracias a planes regionales que prevén determinadas funciones en el territorio; tanto a escala urbana, gracias a cambios del PRG, tal como sucedió en Padua en 2003 (cfr. Ginestri y Passi, 2010), cuando con la modificación parcial del PRG se pusieron en peligro las ZONAS verdes próximas a los barrios poblados, promoviendo un nuevo desarrollo urbano de la ciudad que respondía a una lógica de crecimiento intensivo en las áreas libres.

Las asociaciones ecologistas protestaron recogiendo miles de firmas para defender las «islas» que constituían el «corazón verde» del antiguo PRG, impi-

3. Véase: <http://www.motorcityvr.it/>. 
diendo que se construyera en ellas y para manifestar su disconformidad con algunos proyectos infraestructurales que concernían a la ciudad.

El de Padua es un caso interesante, justamente porque el proyecto abarca la ciudad entera y una parte de su hinterland. Ahora bien, un proyecto de este tipo, que se ha ido delineando a partir de su variante y la elección de algunas actuaciones específicas, por un lado dejan suponer que existe un plan más amplio en el que están implicados actores políticos e institucionales diversos; por otro demuestran que, a pesar de las grandes transformaciones previstas tanto a escala urbana como territorial, no se ha implicado a la población en la toma de decisiones.

Tras las protestas contra el Plan de Ordenación del Territorio Intermunicipal (PATI) del área metropolitana de Padua, se ha encargado un estudio, que va incluido en el Informe Ambiental, sobre las posibles emisiones de $\mathrm{CO}_{2}$ debidas a la realización de las obras planificadas (aumento del tráfico y nuevas parcelaciones productivas y comerciales). Los resultados del estudio, que indican un incremento de las emisiones en la próxima década, no hacen sino reforzar las razones de quien protesta contra la realización de nuevas infraestructuras viarias y nuevas parcelaciones.

En efecto, una de las críticas al PATI se refiere al aumento de superficie edificable sin que haya un plan general para la protección y recalificación del medio ambiente y sin que se hayan puesto en marcha los mecanismos de la participación. Los análisis realizados indican que los PRG de los distintos ayuntamientos han destinado más de $3.000 .000 \mathrm{~m}^{2}$ de áreas todavía inutilizadas en el territorio metropolitano de Padua para actividades productivas, comerciales y direccionales; el PATI, por su parte, ratifica estas decisiones e incluso amplía hasta $1.446 .315 \mathrm{~m}^{2}$ las nuevas áreas de transformación.

También a nivel urbano, a falta de un plan general para la ciudad, se utiliza a menudo la modificación urbanística como instrumento para facilitar transformaciones importantes y a veces radicales, orientadas casi siempre a reducir las áreas verdes.

Muy significativo para el uso y consumo del suelo es el caso de la ribera del Brenta (es decir, un antiguo tramo del río Brenta, hoy en día canal navegable entre Venecia y Padua) (Figura 3), donde están previstos varios proyectos de alto impacto ambiental que las asociaciones locales contestan. El área forma parte de un sistema de asentamientos muy peculiar al que los estudiosos denominan «ciudad difusa», un fenómeno conectado con varios factores de orden económico y social que han ido surgiendo rápidamente a partir de los años setenta y que han determinado un cambio radical en la estructura productiva preexistente convirtiéndola en un distrito de mediana y pequeña empresa. Desde el punto de vista morfológico, el área está sufriendo una gradual urbanización de baja densidad, una configuración territorial que conlleva una serie de problemas en lo referente al consumo del suelo y su permeabilización, además de muchas dificultades a la hora de proporcionar los servicios adecuados a los asentamientos presentes desde hace tiempo.

En el área se concentran varios proyectos, entre los cuales el de Veneto City y el de la Ciudad de la Moda o Verve (Figura 4). En este caso se ha 

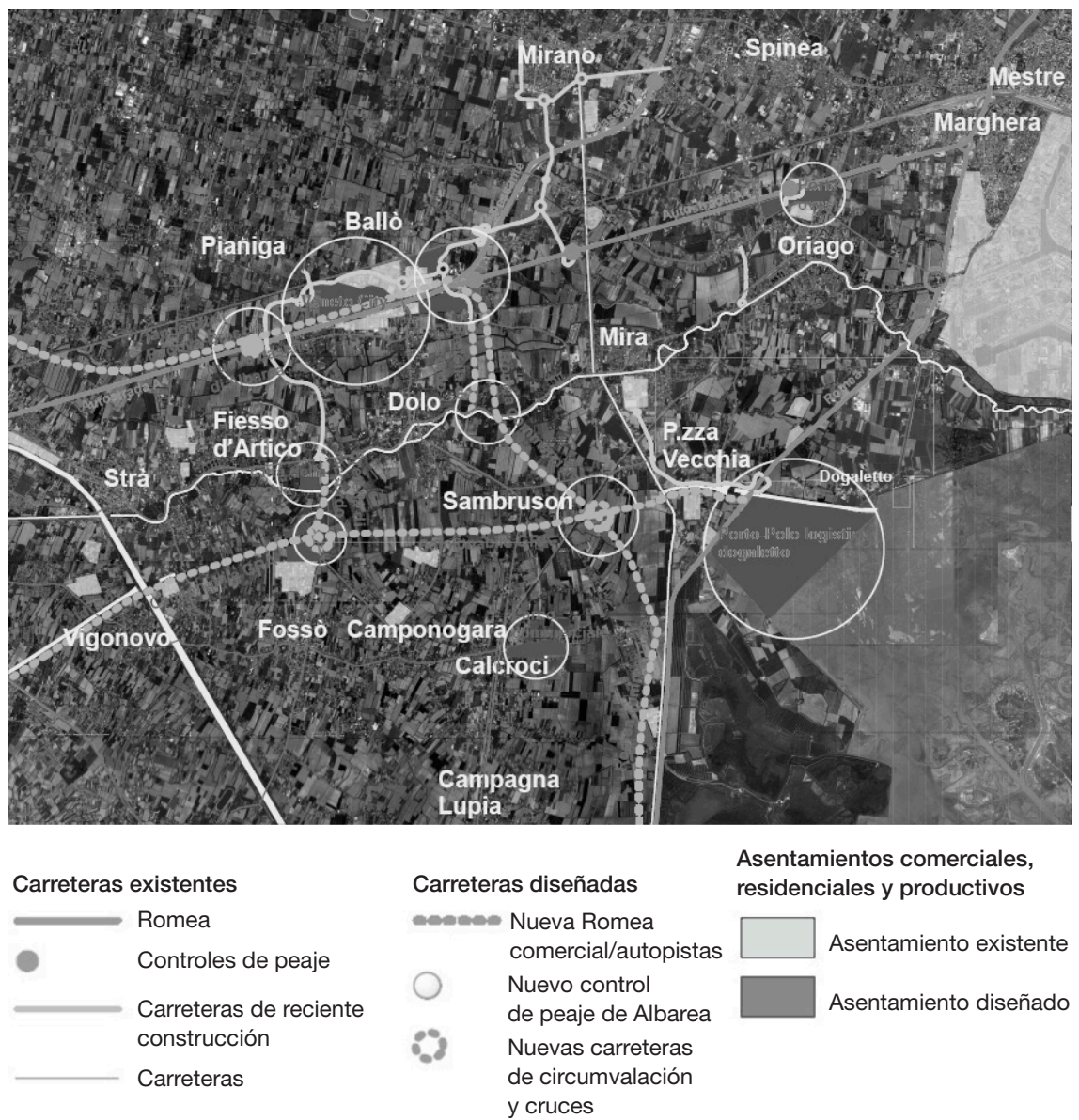

Figura 4. Intervenciones programadas para la Riviera del Brenta (entre Venecia y Padua).

Fuente: Comitati Ambiente Territorio.

elaborado un plan integrado de recalificación urbanística y ambiental, utilizando todo tipo de herramientas para recuperar el espacio urbano y edificado en un área totalmente sin edificar y preeminentemente agrícola.

Verve promueve en concreto obras de edificación residencial, un centro comercial con 70 tiendas de alta moda y también oficinas, hoteles, restaurantes, un centro de congresos, un museo, un teatro, un parque temático y puntos de amarre para embarcaciones en el canal navegable del Brenta, que cubren un total de $115.000 \mathrm{~m}^{2}, 45.000$ de los cuales destinados a la realización del proyecto.

Veneto City (Figura 4), en cambio, cubre una extensión mayor - y con funciones en parte parecidas a las de la Ciudad de la Moda-, situada entre 
los municipios de Dolo y Pianiga y prevé un volumen edificado total de $2.000 .000 \mathrm{~m}^{2}$. El uso y destino del suelo es de tipo mixto, e incluye varios géneros: comercial, direccional regional, equipamiento para la salud, polo ferial, polo universitario, centro de investigación. Veneto City, según entienden sus promotores, debería convertirse en un gran centro multifuncional. Sin embargo, los pueblos de la Ribera han mostrado cierta preocupación por la calidad de vida y el aumento del tráfico junto con el riesgo para el comercio minorista, un problema que ya habían planteado algunas asociaciones profesionales.

Sin duda alguna, el área de Veneto City —el llamado «nudo de Roncoduro»-, tiene una ubicación estratégica por estar cerca de la autopista A4, del ferrocarril y del enlace con el Passante (es decir, la nueva carretera de circunvalación de Mestre); además, no lejos de ella están previstos tanto el enlace con el área denominada Romea Commerciale, como los ramales de acceso al anillo de circunvalación de Padua.

Todo este relato de modificaciones, recalificaciones... y sus efectos no pueden entenderse sin hacer alguna referencia a la evolución del particular sistema infraestructural y de asentamientos de la región para enfocar y comprender a fondo los fenómenos y las opciones más recientes.

En los años setenta y ochenta, el desarrollo y la potenciación del sistema infraestructural de la región respondía sobre todo a la necesidad de realizar obras de urbanización para la expansión de la misma urbanización y de proporcionar servicios a la población que vivía cada día más esparcida por el territorio.

El tejido urbano discontinuo y disperso que se iba formando, y donde no se podía prescindir del coche, presentaba unas cuantas ventajas a nivel individual y a corto plazo se convirtió en un modelo territorial y económico exitoso. A largo plazo, en cambio, han ido surgiendo problemas relacionados con los costes y el derroche de recursos naturales y económicos que este sistema supone.

Las obras infraestructurales, incluso las que se refieren a la viabilidad de la red secundaria, y su realización han contribuido sensiblemente a la creación de una trama urbana dispersa y, en tiempos más recientes, a que las políticas regionales mostraran un renovado interés por las "grandes obras infraestructurales». La Región del Véneto, gracias también al apoyo del gobierno central, desempeña en este sector un papel primario presionando para que se complete el tercer carril de la autopista Venecia-Padua, de la Padua-Brescia y posteriormente de la Venecia-Trieste; ha realizado el anillo de la autopista que circunvala Mestre (el arriba mencionado Passante di Mestre), cuya inauguración data del año 2009; apunta a realizar el anillo de circunvalación de Padua (Grande Raccordo Anulare di Padova), la nueva autovía para el área pedemontana, la parte sur de la A31 Valdastico y la Nueva Romea Comercial (cfr. Fregolent y Savino, 2011), que se inscribe en un proyecto más amplio denominado autopista OrteMestre, que conectaría el mar Tirreno con el Adriático.

En la mayor parte de los casos se trata de carreteras de gran capacidad destinadas al tráfico de largo recorrido que, si por un lado potencian las conexiones viarias del área, por otro no mejoran la movilidad de corta y mediana 
distancia, que se desarrolla según modalidades de tipo incremental sin la más mínima coordinación entre las distintas administraciones. Y lo peor es que se sigue incentivando el transporte por carretera. A estas infraestructuras y a los proyectos para completar tramos ya existentes hay que sumar la realización de otras carreteras de importancia regional, como las circunvalaciones de Verona, Vicenza y Padua o la prolongación de algunas autopistas con sus correspondientes obras complementarias y de conexión.

El resultado es un territorio desgastado y fragmentado que, al estar a la vez muy poblado y «utilizado», necesita que se "remienden los desgarros» producidos, a través de una planificación cuidadosa, orientada a calificar los espacios abiertos, proporcionar servicios y facilitar la movilidad. Servicios y funciones no sólo deben cumplir con las reales exigencias de un territorio, sino que también deben ser el resultado de políticas a escala territorial, capaces de armonizar proyectos e intereses diversos sin perder de vista ni los valores ambientales y paisajísticos ni los derechos de las comunidades allí asentadas; deben ser capaces además de interpretar el "crecimiento» en clave estratégica y sostenible, promoviendo la reutilización de los elementos preexistentes y sobre todo del suelo ya artificializado, para evitar la antropización de más terrenos agrícolas.

Lo dicho nos lleva a abrir una necesaria reflexión sobre las herramientas del plan y sobre el papel que la planificación ha jugado en muchos de los casos contestados. A menudo se eligen localizaciones que responden a intereses particulares y a lógicas lucrativas que acaban desvirtuando el plan como instrumento. Las modificaciones parciales o generales de los planes vigentes sirven justamente para poder intervenir en el territorio sin que haya una estrategia general y sin evaluar previamente los efectos que determinadas opciones van a tener a nivel territorial; en otros casos, en cambio, hay herramientas «sin padre», únicamente creadas porque la ley lo imponía y que en realidad nadie quiere de verdad por ser molestas. Un ejemplo es el de algunos planes ambientales, como el Plan de Gestión de las Zonas de Protección Especial (ZPS) del Delta del Po; instrumentos, creados especialmente para proteger y preservar zonas de frágil equilibrio, que están considerados por los administradores y los ciudadanos implicados como obstáculos para el desarrollo ${ }^{4}$.

En algunos casos, los conflictos que hemos analizado se pueden considerar como el resultado de un «error» en el proyecto o en el plan, debido a localizaciones incorrectas que casi siempre (Bobbio y Zeppetella, 1999) se podían haber evitado, eligiendo opciones diferentes, fruto de una planificación capaz de interpretar el territorio a distintas escalas para luego armonizarlas. Los conflictos a veces se pueden prever y en estos casos lo más importante es intentar evitarlos gracias a prácticas de participación activa con la mediación de comités

4. Es clarificador a este propósito el ejemplo del proyecto para la construcción de una dársena con 700 puntos de atraque, hoteles y áreas comerciales en Porto Caleri, situado en el municipio de Rosolina, que está incluido en una ZPS; ello ha sido posible gracias a una variante del Plan de Área que ya no limita en ningún modo el número de puntos de amarre y que en 2007 no obtuvo el visto bueno de la Comisión Europea por no aplicar la Directiva Habitats. 
y asociaciones ecologistas, mediante negociaciones en las que todas las partes desempeñen un papel importante (Bobbio, 1994; 1996), y también por medio de una profunda evaluación de las peticiones a nivel local, no sólo para admitir todas las propuestas, sino también para extender el diálogo a un abanico más amplio de sujetos (cfr. Cabañas López, Jordi Pinatella y Mercadé Marimon, 2012), diálogo dirigido al establecimiento de una estrategia de desarrollo territorial a pesar de los conflictos existentes (Balducci, 2013).

Hay que implementar acciones compartidas con la participación a nivel local de los diferentes sujetos involucrados, mediante la intervención del «operador" público que puede impulsar la construcción de estructuras flexibles, pero estables, de interacción entre actores institucionales (Tedesco, 2005) para perfeccionar un acuerdo entre los propios actores con respecto a objetivos comunes a raíz de una idea compartida de desarrollo (Barbanente, 2005).

\section{Conclusiones}

La catalogación por fichas y el seguimiento descrito al inicio de este artículo han permitido reunir, además de los datos, unas cuantas informaciones y opiniones críticas de los mismos comités (o de quien ha rellenado las fichas) acerca de otras dinámicas de intervención y transformación del territorio. A la luz de estos datos, el cuadro general se complica aún más, y deben tenerse en cuenta junto con las movilizaciones y los microconflictos no violentos, también las denuncias, las cartas y las protestas menos ruidosas pero igual de importantes.

Sería preciso, por lo tanto, que los comités empezaran a traducir las protestas, los conflictos y el malestar en iniciativas y propuestas para sensibilizar a los órganos institucionales, a quienes les competería gobernar las transformaciones del territorio con propuestas y actuaciones concretas que no puedan prescindir de implicar en el proceso de realización de una obra a todas las partes interesadas.

Para concluir, primero hay que abordar los procesos de transformación territorial de otra manera, implicando más a la población y, segundo, como demuestra el elevado número de fichas relacionadas con los conflictos presentes o ya finalizados, el territorio de la región está tan fragmentado y desgastado que se debe reflexionar muy bien sobre las alternativas posibles para dar solución a los problemas.

Aunque puede que a escala local los concejales y los alcaldes gocen de la confianza de sus electores, el número de comités que surgen de forma espontánea y que manifiestan pacíficamente los intereses de la colectividad demuestra que los ciudadanos no están satisfechos con sus administradores, no se fían de ellos. Esta falta de confianza sólo se puede recuperar implicando e informando más a la gente, mejorando las evaluaciones y aumentando la transparencia durante el planeamiento y la realización de las obras. De esta forma los ciudadanos podrían controlar los procesos en todas sus fases y acercarse poco a poco al complicado aparato burocrático y administrativo. 
Tabla 2. Relación entre comités y sujetos institucionales

\begin{tabular}{cccc} 
& \multicolumn{3}{c}{ Conflicto en curso: ¿los sujetos han implementado formas de diálogo } \\
con los comités?
\end{tabular}

Fuente: <http://mapserver.iuav.it/website/AtlanteMalessereTerritoriale/AtlanteMalessereTerritoriale.html>.

Independientemente de si gozan o no de confianza, podríamos decir que los políticos y los administradores han fracasado, por lo menos parcialmente, porque no han sabido observar, escuchar, reconocer los límites que impone el territorio; no han sabido informar a los ciudadanos ni satisfacer sus necesidades; tampoco aplicar medidas de colaboración y diálogo, tal como demuestran los datos recogidos por medio de la investigación (Tabla 2). (Biorcio, 2007; Vitale, 2007).

Además, tanto el número de comités y grupos de ciudadanos como sus conocimientos sobre las cuestiones ligadas a los conflictos indican que la población está sensibilizada con la temática ambiental y que también ha crecido mucho culturalmente a lo largo de estos años; por consiguiente, necesita una clase política competente y capaz de tomar las decisiones adecuadas para afrontar los complejos factores sociales, económicos y culturales de estos contextos urbanos y territoriales, ya de por sí complicados.

En una situación semejante, se tendrían que incentivar una serie de prácticas para implicar a la población de forma más extendida, lo que hoy acontece sólo en casos esporádicos y en los ayuntamientos más sensibles con este tema. Sin duda es un proceso difícil que necesita tiempo, recursos y energías además de una gran capacidad para coordinar y una gran disponibilidad por parte de todos para llegar a tomar decisiones compartidas incluso si los objetivos a realizar no son modificables. En esto, las asociaciones ecologistas podrían desempeñar un papel importante, mediando en la «negociación» entre ciudadanos y administradores, a favor de las buenas prácticas.

A pesar de que en este contexto la conflictividad creciente exigiría formas de concertación, las decisiones tienden a ser muy polarizadas y contribuyen, en mi opinión, a alimentar los conflictos o incluso a originarlos.

Ello significa que la política no es capaz ni de entender los fenómenos actuales en su complejidad ni de responder a la demanda de los ciudadanos con opciones adecuadas y estratégicas, quizás debido a su preocupación por obtener consenso (cada día más difícil de medir) que la lleva a tomar decisiones parciales y a veces arriesgadas.

En este sentido, el papel del técnico (arquitectos, urbanistas y otros profesionales del sector de la planificación y transformación del territorio) es deter- 
minante; sin embargo, el saber técnico y científico y el asesoramiento de un experto «se está convirtiendo cada día más en una mercancía que se vende y se compra» (Pellizzoni, 2011: 23): las opciones que el decisor elige, sean correctas o incorrectas, acaban por coincidir con las del asesor técnico, independientemente de si existen restricciones territoriales y de si la obra es realmente viable.

Todo esto remite a un problema de responsabilidad más amplio que ya no afecta sólo al político y al administrador, porque sería necesario un cambio cultural mucho más vasto y radical, al cual se dirigen las continuas protestas, la formación de comités y los conflictos, incluso los ocultos. La planificación —o, mejor dicho, el planificador — puede, desde luego, contribuir, de manera contundente, a este cambio, puesto que hay que volver a proponer el mensaje cultural y ético — vehiculado por la planificación — a partir de la urgente necesidad de que «for planning theorists to think further on the issue of planning in a context of conflicting rationalities, recognizing the operation of power as it both shapes and maintains them. There is also a need to find ways of incorporating this understanding into planning education and professional development, in all parts of the world, allowing learners to challenge the normative value systems that are so often routinized in planning practices» (Watson, 3003: 403).

\section{Referencias bibliográficas}

Amistani, Federica (2011). «Expertise e reti socio tecniche. Il caso del rigassificatore di Porto Viro». En: Pellizzoni, Luigi (ed.). I conflitti ambientali. Esperti, politica, istituzioni nelle controversie ecologiche. Bolonia: il Mulino, 149-174.

BaLducci, Alessandro (2013). «Trattare con il nemico: conflitti e trading zone nel gioco del piano». Archivio di studi urbani e regionali, 106, 119-126.

BARBANENTE, Angela (2005). «Sviluppo locale, nuova programmazione e pianificazione territoriale». En Savino, Michelangelo (ed.). Pianificazione alla prova del Mezzogiorno. Milán: FrancoAngeli, 129-145.

BARTOlOMeo, Matteo et al. (2009). Libro Bianco su Conflitti Territoriali e Infrastrutture di Trasporto.

<http://www.trt.it/pubblicazioni.htm> [consulta: 10 de noviembre de 2013].

Biorcio, Roberto (2007). «Democrazia e populismo nella Seconda repubblica». En: M. Maraffi (ed.). Gli italiani e la politica. Bolonia: il Mulino, 187-208.

Biorcio, Roberto (2008). «Partecipazione politica e associazionismo», Partecipazione e conflitto, 0, 67-93.

Bоввіо, Luigi (1994). «La gestione dei tre conflitti ambientali e le possibili alternative». En: IRES (ed.). Di questo accordo lieto. Sulla soluzione negoziale dei conflitti ambientali. Turín: Rosenberg\&Sellier, 11-20, 83-105.

Воввіо, Luigi (1996). «Attori e risorse negli interventi di trasformazione territoriale». En: Bobbio, Luigi (ed.). La democrazia non abita a Gordio. Milán: FrancoAngeli, 64-97.

Bobbio, Luigi y Zeppetella, Alberico (ed.) (1999). Perché proprio qui? Grandi opere e opposizioni locali. Milán: FrancoAngeli.

Воввіо, Luigi (2011). «Conflitti territoriali: sei interpretazioni». TeMA, 4/4, 79-88.

Cabañas López, Néstor; Jordi Pinatella, Moisès y Mercadé Marimon, Montserrat (2012). «Planejament territorial i debat social». En: CASTAÑER, Margarida (ed.). El planejament territorial a Catalunya a inici del segle XX. Barcelona: SCOT, 166-202. 
CARuso, Loris (2010). Il territorio della politica. La nuova partecipazione di massa nei movimenti No Tav e No Dal Molin. Milán: FrancoAngeli.

Castañer Vivas, Margarida; Cabañas Lopez, Néstor y Jordi Pinatella, Moisès (2012). «L'impacte social dels plans i dels projectes territorials. El cas de Catalunya». Documents d'Anàlisi Geogràfica, 58/2, 219-237.

Castells, Manuel (1983). The city and the grassroots: a cross-cultural theory of urban social movements. Londres: Edward Arnold.

Castells, Manuel (2012). Reti di indignazione e speranza. Movimenti sociali nell'era di Internet. Milán: Università Bocconi Editore.

Cruz Gallach, Helena (2009). «I conflitti territoriali in Catalogna: verso una caratterizzazione delle mobilitazioni sociali esistenti». Archivio di studi urbani e regionali, 95, 59-76.

Cruz Gallach, Helena y Martí Costa, Marc (2010). "Conflictos urbanísticos y movilizaciones ciudadanas: reflexiones desde Barcelona». Finisterra, 90, 111-131.

Della Porta, Donatella (2008). «Eventful Protest, Global Conflicts». Distinktion: Scandinavian Journal of Social Theory, 9(2), 27-56.

Della Porta, Donatella y Diani, Mario (1997). I movimenti sociali. Roma: Carocci.

- (2004). Movimenti senza protesta? L'ambientalismo in Italia. Bolonia: il Mulino.

Della Porta, Donatella y Tarrow, Sidney (eds.) (2005). Transnational Protest and Global Activism. Nueva York: Rowman \& Littlefield, 21-43.

Fainstein, Susan (2005). "Planning theory and the city». Journal of Planning Education and Research. 25, 121-130.

Fedi, Angela y Mannarini, Terri (ed.) (2008). Oltre il Nimby. La dimensione psico-sociale della protesta contro le opere sgradite. Milán: FrancoAngeli.

Fregolent, Laura y SAvino, Michelangelo (2011). «Quando la soluzione diventa un nuovo problema. Prospettive della dispersione veneta tra politiche infrastrutturali e consumo di suolo». En: Pezzagno, Michèle y Docchio, Silvia (ed.). Atti della XVII Conferenza Internazionale From metropolitan city to metropolitan corridor: the case of the Po Valley Corridor. Forlì: Egaf edizioni, 263-278.

Ginestri, Sandro y Passi Lucio (ed.) (2010). Il danno. Padova: verde, speculazione e cemento nella seconda Repubblica. Legambiente. <www.legambientepadova.it/files/Il_Danno_web.pdf>. [consulta: 25 de enero de 2012].

Mannarini, Terri; Bonomelli, Ronnie y Caruso, Loris (2008). «Il rapporto con la natura e il territorio». En: Fedi, Angela y Mannarini, Terri (ed.). Oltre il Nimby. La dimensione psico-sociale della protesta contro le opere sgradite. Milán: FrancoAngeli, 97-123.

Muñiz i SAn Martín, Sigrid (2012). «Conflictividad ambiental y territorial: un área de investigación en construcción. Aproximaciones al caso catalán». Documents d'Anàlisi Geogràfica, 58(3), 481-496.

NeL.Lo, Oriol (2003). Aquí, no! Els conflictes territorials a Catalunya. Barcelona: Editorial Empúries.

Nogué, Joan y Wilbrand, S. (2010). «Landscape, territory, and civil society in Catalonia». Environment and Planning D, 28(4), 638-652.

Osti, Giorgio (2011). «Dalla terra al mare. Esperti e istituzioni nella realizzazione di una grande infrastruttura energetica». En: Pellizzoni, Luigi (ed.). I conflitti ambientali. Esperti, politica, istituzioni nelle controversie ecologiche. Bolonia: il Mulino, 175-197.

Pellizzoni, Luigi (ed.) (2011). I conflitti ambientali. Esperti, politica, istituzioni nelle controversie ecologiche. Bolonia: il Mulino. 
Rootes, Christopher (2005). «A limited Transnationalization?: The British Environmental Movement». En: Della Porta, Donatella y Tarrow, Sidney (eds.). Transnational Protest and Global Activism. Nueva York: Rowman \& Littlefield, 21-43.

Rosanvallon, Pierre (2006). "La contro-democrazia». Ricerche di storia politica. 3, 289-301.

Societat Catalana d'Ordenació del Territori (SCOT) (2007). Anuari Territorial de Catalunya. Barcelona: Societat Catalana d'Ordenació del Territori.

Tedesco, Carla (2005). «Partecipare (come e perché) alle politiche urbane? Un caso di studio in Puglia». En: Gelli, Francesca (ed.). La democrazia locale tra rappresentanza e partecipazione. Milán: FrancoAngeli, 201-215.

Varotto, Mauro y Visentin, Ludovico Fabrizio (2008). «Comitati locali e criticità ambientali in Veneto. L'evoluzione del fenomeno negli ultimi 10 anni». ARS, $116,9-17$.

Vitale, Tommaso (2007). «Le tensioni tra partecipazione e rappresentanza e i dilemmi dell'azione collettiva nelle mobilitazioni locali». En: Vitale, Tommaso (ed.). In nome di chi? Partecipazione e rappresentanza nelle mobilitazioni locali. Milán: FrancoAngeli, 9-40.

Zamparutti, Antony (ed.) (2000). «Difendere l'ambiente nel Veneto: conflitti e comitati locali». Quaderno, 3. Verona: Osservatorio Veneto.

Watson, Vanessa (2003). "Conflicting Rationalities: Implications for Planning Theory and Ethics». Planning Theory \& Practice, 4/4, 395-407. 